\title{
RESONANCE BEHAVIOR OF LANGANITE AND LANGATATE PLATES DRIVEN BY LATERAL FIELD EXCITATION
}

\author{
Yoonkee Kim, Ajmal Khan', and Arthur Ballato, US Army CECOM, Fort Monmouth, NJ
}

\begin{abstract}
Langanite ( $\mathrm{LGN}, \mathrm{La}_{3} \mathrm{Ga}_{5.5} \mathrm{Nb}_{0.5} \mathrm{O}_{14}$ ), Langatate (LGT, $\mathrm{La}_{3} \mathrm{Ga}_{5.5} \mathrm{Ta}_{0.5} \mathrm{O}_{14}$ ) single-crystal plano-convex resonators were driven in thickness modes by lateral field excitation using a custom fixture. Resonance behavior was characterized as a function of in-plane (azimuthal) rotation angle for the thickness vibration modes of doubly rotated orientation. Specifically, $S_{11}$ reflection coefficients were measured for the first and third overtones of the c-mode and the b-mode using a network analyzer and converted into relative piezoelectric coupling coefficients. Results are presented in terms of relative lateral piezoelectric coupling coefficients versus azimuthal angle. Comparison with theoretical predictions, made for the case of simple thickness modes (i.e., plane waves) using an extension of the Christoffel-Bechmann method with published materials constants, shows good agreement. Furthermore, it is demonstrated that lateral field excitation of some doubly rotated cuts of LGN and LGT offers the potential for significantly enhancing c-mode piezoelectric coupling, and for tuning out the unwanted b-mode, as compared to thickness field excitation.
\end{abstract}

\section{Introduction}

Single crystals of langanite $\left(\mathrm{La}_{3} \mathrm{Ga}_{5.5} \mathrm{Nb}_{0.5} \mathrm{O}_{14}\right.$ or $\left.\mathrm{LGN}\right)$ and langatate $\left(\mathrm{La}_{3} \mathrm{Ga}_{5.5} \mathrm{Ta}_{0.5} \mathrm{O}_{14}\right.$ or LGT) are of considerable interest for both bulk acoustic wave and surface acoustic wave devices $[1,2,3]$. This is due to a combination of a moderately large piezoelectric coupling factor, moderate dielectric permittivity, very small acoustic loss (high $Q$ ) and temperature-compensated orientations for thickness-shear waves ( $c$ mode). These materials are of particular interest because they are expected to perform better than quartz in certain frequency control applications.

The $Q$ values for LGN and LGT are particularly impressive in comparison to quartz as reflected in $Q$.frequency $(Q \cdot f)$ products as large as $29 \times 10^{6}$, where $f$ is in $\mathrm{MHz}$, for unplated Y-cut plano-convex LGN [4] and LGT [3] resonators driven by thickness-field-excitation (TFE). To put these values in perspective, consider that the upper bound $Q f$ for AT-cut natural quartz resonators is $15 \mathrm{x}$ $10^{6}[5]$.

\footnotetext{
' Now with Advanced Cerametrics Inc., Lambertville, NJ.
}

The various acoustic modes are usually driven in a piezoelectric crystal plate by TFE where the electric field direction is in the plate-thickness direction. This is achieved by placing the electrodes on opposing plate faces as shown in Figure 1A. Alternatively, a piezoelectric plate can be driven by lateral-field-excitation (LFE) where the electric field is in the plane of the plate. This can be achieved by placing the electrodes on the same face of the plate, as shown in Figure 1B. The angle $\psi$ indicates the direction of the driving electric field (E-field), with respect to the crystallographic axes of the plate (often referred to as the Efield azimuth angle).

LFE offers a number of advantages over TFE as have been previously outlined by Ballato, et al. [6]. For example LFE can result in reduced aging, higher $Q$ values and increased frequency stability, because the regions of greatest vibrational motion are free of electrodes. Additionally, undesired modes that are unavoidable for TFE of a plate of given orientation can be eliminated by varying the E-field azimuth angle, $\psi$, for LFE (e.g., the $b$ mode in SC-cut quartz [7]). LFE also enables certain plate modes that are not available by TFE to be driven. LFE of LGN and LGT is of particular interest since like langasite $\left(\mathrm{La}_{3} \mathrm{Ga}_{5} \mathrm{SiO}_{14}\right.$ or LGS) they are expected to exhibit low impedance levels when driven by TFE as noted by Détaint, et al. $[8,9]$.

(A)

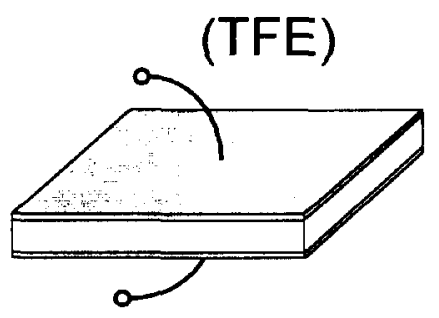

(B)

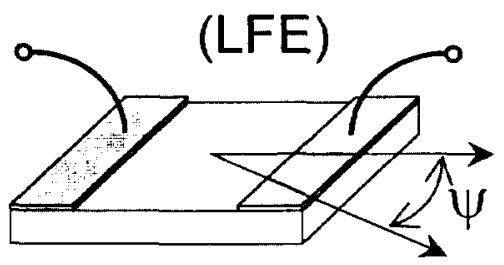

Figure 1: Excitation geometries: (A) thickness-field excitation (TFE) and (B) lateral-field-excitation (LFE). 
For the above reasons a study of LFE of LGN and LGT is highly desirable. Previously, theoretical piezoelectric coupling factor vs. azimuthal angle curves for various LGN and LGT cuts were determined using an extension of the Christoffel-Bechmann method [10]. This paper presents experimental data for select LGN and LGT resonators driven by LFE and is intended to complement the earlier study and to provide more insight into LFE of resonators of these materials.

\section{Experimental Procedure}

Table 1 shows the specifications of the LGN and the LGT material investigated for this study. Several samples of each material were measured, and interesting results were chosen for discussion in this paper.

Instead of depositing electrodes directly on the surface of the sample, a simple LFE fixture shown in Figure 2 was fabricated using a copper-plated PC board. The fixture allowed arbitrary azimuthal angles to be obtained by simply rotating the sample. The electrode gap was $\sim 1 \mathrm{~mm}$. The asperities at the edges of the electrodes were minimized to make the edges smooth thus resulting in uniform distribution of the electric field. Each sample was placed on the fixture with its contoured side facing up and flat side facing the electrodes. The edges of the flat side were supported on a pair of $0.1 \mathrm{~mm}$ thick paper shims thus providing an air gap to eliminate friction between the active region of the sample and the electrodes. Since the samples were contoured, the center of each sample was carefully placed above the center of the electrode gap to obtain the maximum energy coupling at each azimuthal angle. Such placement was critical for accurate reading of coupling factors. The samples were oriented using a reference flat perpendicular to the crystallographic $\mathrm{x}$-axis that was machined into each sample and the azimuthal angle was measured with a protractor.

The reflection coefficient, $S_{11}$, of each sample was measured at laboratory ambient temperature with a network analyzer. The frequency at the base of the valley of an $S_{11}$ spectrograph represents a mode frequency. The square root of the magnitude of $S_{11}$ at the base of the valley from the baseline (which is $0 \mathrm{~dB}$ when the network analyzer is calibrated) of the $S_{11}$ spectrograph is proportional to the coupling factor since the admittance of a resonator is proportional to the square of the coupling factor. After taking the data as a function of the azimuthal angle, uniform scaling was applied to the data to see if the relative profile matched the theoretical curve.

Possible sources responsible for the offset observed between the experimental data and the theoretical curve include: (1) measurement error (e.g., centering of the contoured sample onto the electrode gap, aligning and reading of the protractor $\delta \psi$, and measurement noise), (2) sample fabrication error (e.g., error in reference flat of samples $\Delta \psi$, error in cut angle, $\Delta \phi$ and $\Delta \theta$ ), and (3) accuracy of material constants used to determine the theoretical curve.

\section{Sensitivity Analysis}

By comparing the LFE experimental curves with the theoretical curves, we may be able to confirm the accuracy of the published material constants. We conducted a sensitivity analysis for this purpose.

Piezoelectric coupling factors, $k$, for the three simple thickness vibration modes (quasi-extensional, $A$, fast-quasishear, $B$, and slow-quasi-shear, $C$ ) of single-crystal piezoelectric plates driven by LFE were calculated by assuming standing plane acoustic waves propagating in a piezoelectric material in a specific direction with respect to the crystallographic axes and solving the appropriate wave equations. The equations were solved using the ChristoffelBechmann (C-B) method, extended for LFE, as described previously by Ballato $[11,12]$. The $C-B$ method was used rather than the alternative plate-coordinate method since it is computationally simpler [12].

Calculations for LGN were made using the material constants of Pisarevsky, et al. [13]. Calculations for LGT were made using the piezoelectric constants reported by Smythe and Hague [14] and the elastic and dielectric constants reported by Malocha, et al. [15] as part of a joint effort. (The elastic, piezoelectric and dielectric matrices were the same as for $\alpha$-quartz since LGN and LGT also belong to the trigonal, 32 crystal class [16].)

Table 1: Materials investigated.

\begin{tabular}{|c|c|c|}
\hline Material & LGN & LGT \\
\hline Orientation & $\left(\phi=23.5^{\circ}, \theta=35^{\circ}\right)$ & $\left(\phi=30^{\circ}, \theta=45^{\circ}\right)$ \\
\hline Diameter & $14 \mathrm{~mm}\left(0.550^{\prime \prime}\right)$ & $14 \mathrm{~mm}\left(0.550^{\prime \prime}\right)$ \\
\hline Contour & 4 diopter, plano-convex & 2 diopter, plano-convex \\
\hline C1 mode & $2.515 \mathrm{MHz}$ & $1.972 \mathrm{MHz}$ \\
\hline
\end{tabular}

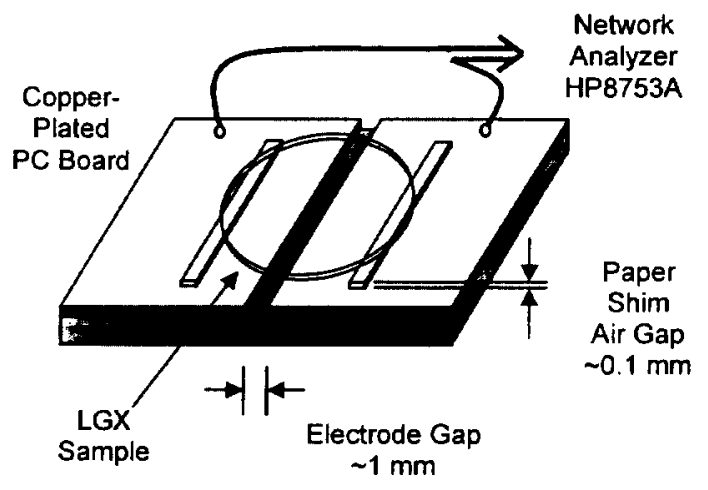

Figure 2: LFE fixture. 
The system of notation used for designating the crystallographic orientation of a given plate, and the orientation of the E-field relative to the plate crystal axes, is illustrated in Figures $3 \mathrm{~A}$ and $3 \mathrm{~B}$, respectively. The two rotation angles $\phi$ and $\theta$, taken successively around the $z$ and $x$ (or $x^{\prime}$ ) axes, respectively, describe the plate orientation (Fig. 3A); The final rotation $\psi$, taken around the normal of the oriented plate, with $\psi=0^{\circ}$ corresponding to the $x$ (or $x^{\prime}$ ) axis, represents the E-field direction. The piezoelectric coupling factors $k$ for the $A, B$ and $C$ mode driven by LFE were determined as a function of $\psi$. Results are presented as plots of $k$ versus $\psi$ for the range $-90^{\circ}$ to $+90^{\circ}$.

Each of $c_{11}, c_{44}$, and $c_{66}$ was changed individually by $\pm 5 \%$ to see the dependency of the coupling factors on each stiffness constant. The coupling factors were calculated as a function of the azimuthal angle $\psi$ with a $1^{\circ}$ step size (i.e., $\left.90^{\circ},-89^{\circ}, \ldots, 90^{\circ}\right)$. The results are shown in Figures 4 and 5 for the investigated cuts of LGN and LGT, respectively. We need to consider the shift of $\psi$ where the coupling factors are zero since it is the most practical to measure experimentally, i.e., is the most distinguishable. The interpolated shifts are summarized in Table 2 . Note that the smallest shift angles, i.e., $<1^{\circ}$ are not meaningful because they are below the cut and measurement tolerances. This analysis reveals that $c_{11}$ is the most sensitive of the three constants for both materials. Further, mode sensitivity to

(A)

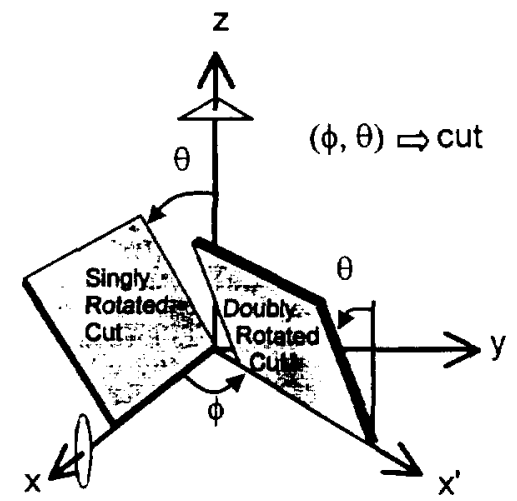

(B)

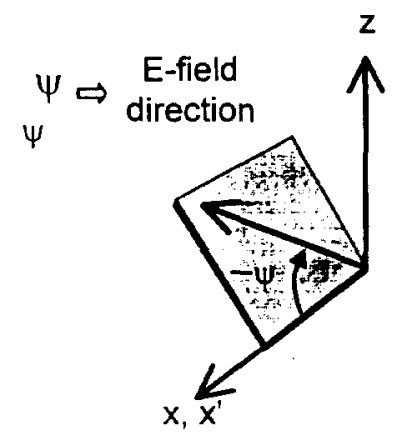

Figure 3: Notation system used for designating (A crystallographic orientation of a given plate, and (B) Efield direction relative to the crystallographic axes. changes in $c_{11}$ is the greatest for the $B$ mode, moderate for the $\mathrm{C}$ mode and the least for the $A$ mode.

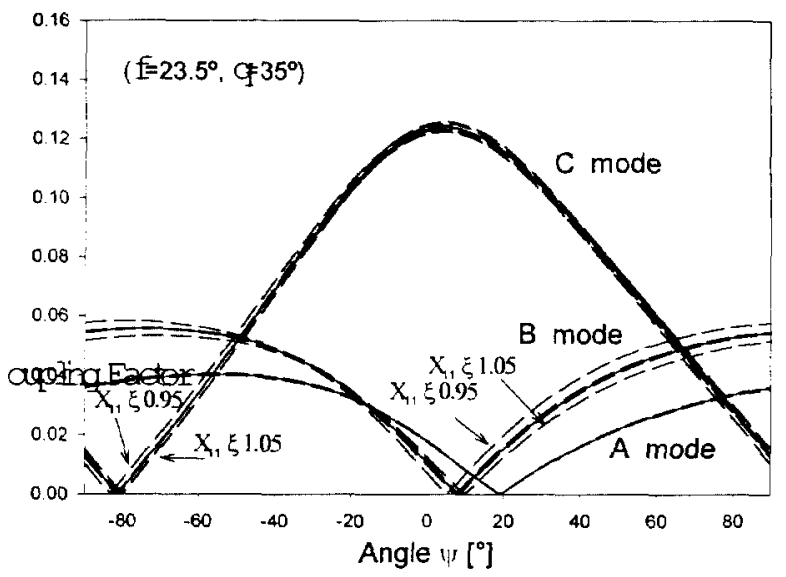

Figure 4: Theoretical sensitivity analysis of LGN with individual changes of $c_{11}, c_{44}$, and $c_{66}$ each by $\pm 5 \%$.

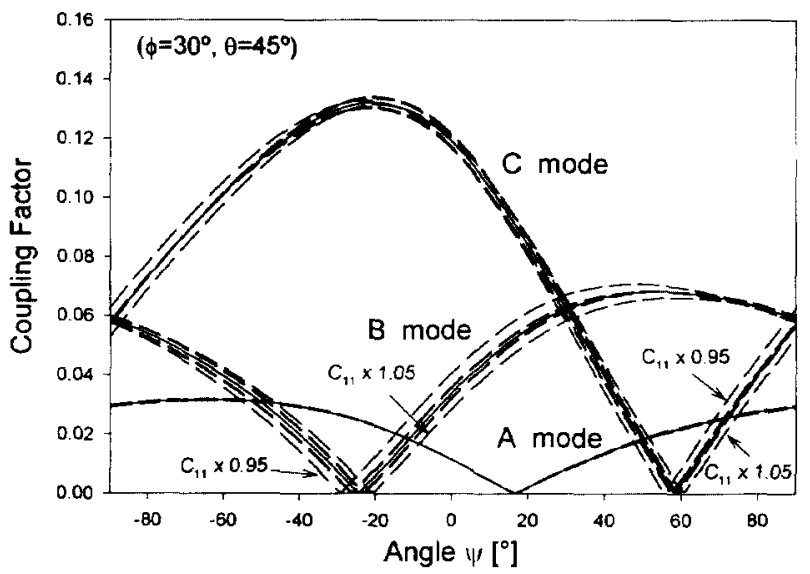

Figure 5: Theoretical sensitivity analysis of LGT with individual changes of $c_{11}, c_{44}$, and $c_{66}$ each by $\pm 5 \%$.

Table 2: The calculated azimuthal angle shift of each mode for the investigated cuts of LGN and LGT where $k=0$. Each of $c_{11}, c_{44}$, and $c_{66}$ was changed individually by $\pm 5 \%$.

\begin{tabular}{|c|c|c|c|c|c|c|}
\hline Material & \multicolumn{2}{|c|}{$\operatorname{LGN}\left(\phi=23.5^{\circ}, \theta=35^{\circ}\right)$} & \multicolumn{3}{c|}{ LGT $\left(\phi=30^{\circ}, \theta=45^{\circ}\right)$} \\
\hline Mode & $C$ & $B$ & $A$ & $C$ & $B$ & $A$ \\
\hline $\mathrm{c}_{11}$ & $3.2^{\circ}$ & $4.7^{\circ}$ & $0.1^{\circ}$ & $4.9^{\circ}$ & $8.3^{\circ}$ & $0.4^{\circ}$ \\
\hline $\mathrm{c}_{44}$ & $0.5^{\circ}$ & $1.0^{\circ}$ & $0.1^{\circ}$ & $1.7^{\circ}$ & $3.5^{\circ}$ & $0^{\circ}$ \\
\hline $\mathrm{c}_{66}$ & $0.7^{\circ}$ & $1.1^{\circ}$ & $0.1^{\circ}$ & $1.4^{\circ}$ & $2.9^{\circ}$ & $0.1^{\circ}$ \\
\hline
\end{tabular}




\section{Results and Discussion}

The calculated and measured mode frequencies of a sample are shown in Table 3 . Strong responses at the fundamental and the $3^{\text {rd }}$ overtone modes were selected for measurement and many weakly coupled anharmonic modes were ignored. Since the sample was contoured, the measured $\mathrm{Cl}$ mode frequency was also used for the theoretical value of $C l$ mode and the theoretical $C 3$ and $B$ mode frequencies were obtained using Stevens-Tiersten theory $[17,18]$ to include the resonator contour in the calculation. The calculated eigenmode velocities of the investigated cuts of LGN and LGT are $(A$ mode $=5804.68$ $\mathrm{m} / \mathrm{s}, B$ mode $=3252.95 \mathrm{~m} / \mathrm{s}, C$ mode $=2808.59 \mathrm{~m} / \mathrm{s})$ and $(A$ $\operatorname{mode}=5962.55 \mathrm{~m} / \mathrm{s}, B$ mode $=3155.56 \mathrm{~m} / \mathrm{s}, C$ mode $=$ $2619.26 \mathrm{~m} / \mathrm{s}$ ), respectively. The $\mathrm{Cl}$ and $\mathrm{C} 3$ modes were measurable for both materials. Note that the $C 3$ mode of the LGT sample is in excellent agreement with the theoretical value. The $B 3$ mode of the LGT sample was not measurable and neither $B l$ nor $B 3$ mode of the LGN sample was measurable. It is not clear why the $B 1$ mode of the LGN sample was not strong enough to be measurable. The reason why the $A$ modes were not measurable is probably because the sample is contoured.

Table 3: Comparison of theoretical and experimental mode frequencies in $\mathrm{MHz}$.

\begin{tabular}{|c|c|c|c|c|}
\hline Material & \multicolumn{2}{|c|}{ LGN } & \multicolumn{2}{c|}{ LGT } \\
\hline Mode & Theo. & Exp. & Theo. & Exp. \\
\hline$C I$ & 2.515 & 2.515 & 1.972 & 1.972 \\
\hline$B I$ & 2.906 & - & 2.367 & 2.395 \\
\hline$C 3$ & untrapped & 7.376 & 5.813 & 5.812 \\
\hline$B 3$ & untrapped & - & untrapped & - \\
\hline
\end{tabular}

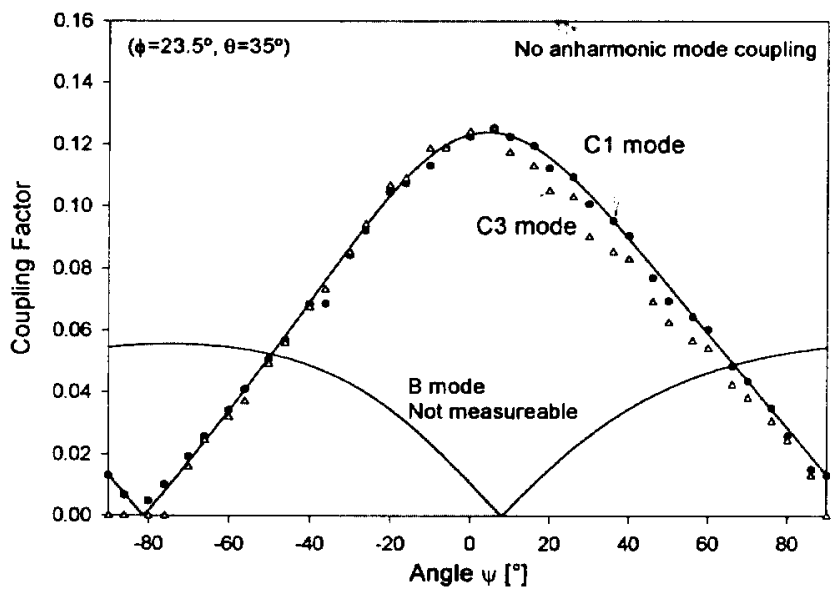

Figure 6: Coupling factors of LGN: lines show theoretical values and symbols show relative profiles obtained from measured data.
Figure 6 shows comparison of the theoretical coupling factor curves and experimental data as a function of angle $\psi$ of an LGN sample. The lines are theoretical curves and the symbols are measured data of the $C l$ and $C 3$ modes. Each of the $\mathrm{Cl}$ and the $\mathrm{C} 3$ modes of this particular sample showed a single "valley" for the $S_{11}$ response without any interfering modes near the valley. The measured data show a good match with the theoretical curves. However, another LGN sample had an interfering mode at the $C l$ mode as in the LGT sample explained below while there was no interfering mode at the $C 3$ mode.

Figure 7 shows the coupling factors of an LGT sample. The lines are theoretical curves and the symbols are measured data of the $C 1, C 3$, and $B 1$ modes. The two inserted graphs show the responses of the $\mathrm{Cl}$ and the $C 3$ modes measured at $\psi=-30^{\circ}$. While the $C 3$ mode showed a single valley for the $S_{11}$ response, without any interfering modes as $\psi$ was varied, the $C l$ mode had two valleys due to two interfering modes with only $\sim 20 \mathrm{ppm}$ separation over $\psi=-60^{\circ} \sim 30^{\circ}$. From $\psi=-60^{\circ}$, the interfering mode appears and its magnitude becomes stronger as the angle approaches $-21^{\circ}$ where the coupling factor reaches a theoretical maximum value. As the angle increases past $21^{\circ}$, the magnitude of the interfering mode decreases and finally disappears above $\sim 30^{\circ}$. The exact angles at which the interfering mode appeared were not easily discernible from the $S_{11}$ spectrograph. The mode separation frequency also changes as the angle changes. When such an interfering mode exists, the acoustic energy is divided into two modes, and the relative profile of the experimental coupling factor doesn't match well with the theoretical one.

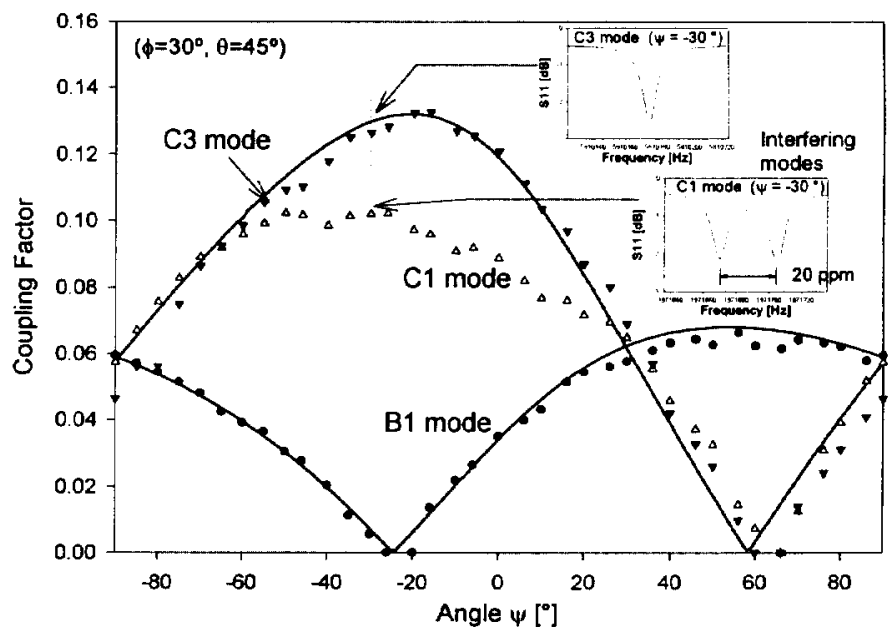

Figure 7: Coupling factors of LGT: lines show theoretical values and symbols show relative profiles obtained from measured data. 
This explains the poor match between the experimental data and the theoretical curve for the $\mathrm{C} 1$ mode for $-21^{\circ}<\psi<$ $-30^{\circ}$ in Fig. 7. The $B l$ mode profile also shows that a weakly interfering mode exists as indicated by the ripples of the coupling factor for $\psi=20^{\circ} \sim-80^{\circ}$, and this is consistent with the existence of the interfering mode at the $\mathrm{Cl}$ mode. The existence of an interfering mode at the $B I$ mode was much more obvious in another LGT sample. The difference in response from sample-to-sample is presumably caused by slight sample-to-sample variations, e.g., variations in the surface finish, the mismatch between the contour apex and the center of the sample, and parallelism of the faces of the starting resonator blanks. For the $C 3$ mode, the experimental profile matches well with the theoretical curve because no interfering mode was present. From these observations, we may conclude that a diopter of 2 for LGT is good to make a C3 mode LFE resonator but not a $\mathrm{Cl}$ mode one. Note that there is a slight shift of the azimuthal angle where zero coupling occurs between the experimental curve and the theoretical one. The possible causes of this shift are discussed in the Experimental Procedure section.

Perhaps the most notable features of the LFE coupling plots shown in Figs. 6 and 7 are the potential for eliminating unwanted modes and for achieving greater coupling magnitudes versus TFE by appropriate adjustment of $\psi$. These features are particularly significant when we consider that a doubly rotated cut of LGN with similar rotation angles, i.e., $\phi=26.9^{\circ}, \theta=43.26^{\circ}$, has been recently predicted to be temperature compensated for the $\mathrm{C}$ mode by da Cunha, et al. [19]. Specifically, they predicted a cubic frequency change versus temperature response for the $C$ mode around room temperature for this cut making it of potential practical interest. Figure 6 shows the predicted $k_{\mathrm{m}}(\mathrm{LFE})$ versus $\psi$ plots (where $\mathrm{m}$ refers to the mode) for a similar LGN cut. The maximum $k_{\mathrm{c}}$ (LFE) achievable $(\approx 12.4$ $\%$ at $\psi=4^{\circ}$ ) is considerably greater than $k_{\mathrm{c}}$ (TFE) $(\approx 1.0 \%$ ). Additionally, either the $A$ or $B$ mode can be eliminated by adjusting $\psi$ appropriately; This may be a considerable advantage of LFE versus TFE for some doubly rotated cuts of LGN considering that for TFE both the $A$ and $B$ mode couple more strongly than the $B$ mode. This advantage also applies to some doubly rotated cuts of LGT such as that shown in Figure 7. Similar to the LGN case, the maximum $k_{\mathrm{c}}$ (LFE) achievable $\left(\approx 13.2 \%\right.$ at $\left.\psi=-21^{\circ}\right)$ in the LGT is considerably greater than $k_{\mathrm{c}}$ (TFE) $(\approx 2.8 \%$ ).

\section{Conclusions}

Piezoelectric coupling factors for the thickness vibration modes driven by LFE were measured as a function of electric field azimuth angle, $\psi$, for doubly rotated cuts of LGN and LGT. The experimental data matched very well with the theoretical values calculated using the Christoffel-Bechmann method with published materials constants. The results showed that the coupling measurements were consistent with published material values. The ability to eliminate unwanted modes and to achieve greater coupling for the $\mathrm{C}$ mode by LFE versus TFE of doubly rotated cuts of LGN and LGT, that are of potential practical interest, was demonstrated.

\section{Acknowledgments}

The authors wish to thank R. C. Smythe, Piezo Technology, Inc., Orlando, FL, for providing the theoretical mode frequency calculation and the samples investigated in this paper.

\section{References}

1. T. Fukuda, H. Takeda, K. Shimamura, H. Kawanaka, M. Kumatoriya, S. Murakami, J. Sato and M. Sato, "Growth of new langasite single crystals for piezoelectric applications," Proc. of the Eleventh IEEE Int'l. Symp. on Applications of Ferro, Montreux, Switzerland, pp. 315-319, 1998.

2. M. Kadota, J. Nakanishi, T. Kitamura and M. Kumatoriya, "Surface acoustic wave properties on rotated Y-cut langasite single crystal substrates," Proc. of the Eleventh IEEE Int'l. Symp. on Applications of Ferro, Montreux, Switzerland, pp. 357-360, 1998.

3. R. C. Smythe, R. C. Helmbold, G. E. Hague and K. A. Snow, "Langasite, langanite and langatate bulk-wave Y-cut resonators," IEEE Trans. Ultrason. Ferro. and Freq. Contr. vol. 47 , no. 2 , pp. $355-360,2000$

4. "Acceleration Insensitive Clocks," Report on Contract No. N66001-97C-8634, March 2000, General Technical Services, Wall, NJ 07719.

5. A. W. Warner, "Design and performance of ultraprecise 2.5me quartz crystal units," Bell Syst. Tech. J, vol. 39, no. 9, pp. 1193-1217, 1960.

6. A. Ballato, E. R. Hatch, M. Mizan and T. Lukaszek, "Lateral Field Equivalent Networks and Piezocoupling Factors of Quartz Plates Driven in Simple Thickness Modes," IEEE Trans. Ultrason. Ferro and Freq. Contr, vol. UFFC-33, no. 4, pp. 385-393, 1986.

7. E. R. Hatch and A. Ballato, "Lateral-field excitation of quartz plates," Proc. IEEE Ultrason. Symp., pp. 512-515, 1983

8. J. Détaint, J. Schwartzel, A. Zarka, B. Capelle, J. P. Denis and E. Philippot, "Bulk wave propagation and energy trapping in the new thermally compensated materials with trigonal symmetry," Proc. IEEE Int'l. Freq. Con. Symp., pp. 58-71, 1994.

9. J. Détaint, B. Capelle, A. Zarka and D. Cochet-Muchy, "Langasite plano-convex resonators using lateral or perpendicular field excitation." Proc. Eur. Freq. Time For., pp. 289-296, 1995.

10. A. Khan and A. Ballato, "Lateral Field Excitation Predictions for Plates of Langasite and Isomorphs Driven in Simple Thickness Modes," Proc. 2000 IEEE/EIA Int'l. Freq. Con. Symp., pp. 180-185, 2000

11. A. Ballato, "Lateral and thickness excitation of obliquely poled ferroelectric ceramic plates," Ceramic Transactions, Vol. 106, pp. 309-332, 2000. ISBN 1-57498 098-X. 
12. A. Ballato, "Extended Christoffel-Bechmann elastic wave formalism for piezoelectric, dielectric media," Proc. 2000 IEEE/EIA Int'l. Freq. Con. Symp., pp. 340-344, 2000.

13. Yu. V. Pisarevsky, P. A. Senushencov, P. A. Popov and B. V. Mill, "New strong piezoelectric $\mathrm{La}_{3} \mathrm{Ga}_{5.5} \mathrm{Nb}_{0.5} \mathrm{O}_{14}$ with temperature compensation cuts," Proc. IEEE Int'l. Freq. Con. Symp., pp. 653-656, 1995.

14. R. C. Smythe and G. E. Hague, "Determination of the piezoelectric constants of LGN, LGS and LGT," Proc. 2000 IEEE/EIA Int'l. Freq. Con. Symp., pp. 191-194, 2000.

15. D. C. Malocha, M.P. da Cunha, E. Adler, R. C. Smythe, S. Fredric, M. Chou, R. Helmbold and Y. S. Zhou, "Recent measurements of material constants versus temperature for langatate and langanite," Proc. 2000 IEEE/EIA Int'l. Freq. Con. Symp., pp. 200-205, 2000.

16. B. V. Mill, A. V. Butashin, G. G. Khodzhabagyan, E. L. Belokoneva and N. V. Belov, "Modified rare-earth gallates with a $\mathrm{Ca}_{3} \mathrm{Ga}_{2} \mathrm{Ge}_{4} \mathrm{O}_{14}$ structure," Sov. Phys. Dokl., vol. 27, no. 6 , pp. 434-437, 1982.

17. D.S. Stevens \& H.F. Tiersten, "An Analysis of DoublyRotated Contoured Quartz Crystal Resonators," Proc. 39th Annual Frequency Control Symp., pp.436-447, 1985.

18. D.S. Stevens \& H.F. Tiersten, "An Analysis of Doubly. Rotated Quartz Crystal Resonators Using Essentially Thickness Modes with Transverse Variation," J. Acoust. Soc. Am., v.79, pp.1811-1826, 1986.

19. M. P. da Cunha, E. L. Adler and D. C. Malocha, "BAW temperature sensitivity and coupling in langanite," Proc. IEEE Int'l. Ultrason. Symp., pp. 883-886, 1999. 